\title{
Energy resolved ultrafast relaxation dynamics close to the band edge of low-temperature grown GaAs
}

\author{
G. Segschneider, ${ }^{\text {a) }}$ T. Dekorsy, and H. Kurz \\ Institut für Halbleitertechnik II, Rheinisch-Westfälische Technische Hochschule (RWTH) Aachen, \\ Sommerfeldstr. 24, D-52056 Aachen, Germany \\ R. Hey and K. Ploog \\ Paul-Drude-Institut für Festkörperphysik, Hausvogteiplatz, 5-7, D-10117 Berlin, Germany
}

\begin{abstract}
We investigate the relaxation dynamics of photogenerated carriers in low-temperature grown GaAs by femtosecond pump-probe measurements. The carrier dynamics in the vicinity of the band edge is disentangled in a two-color technique. The filling of shallow bound states close beneath the band edge is resolved. A temporal delay in the occupation of these states as well as a large optical nonlinearity points towards microscopic potential fluctuations forming these states.
\end{abstract}

In the last few years the interest in low-temperature grown (LT) semiconductors rapidly increased because of their unique electrical and optical properties and the resulting wide range of optoelectronic applications. The most promising material is LT GaAs grown at temperatures $T_{g}=180^{\circ}-300^{\circ} \mathrm{C}$ by molecular beam epitaxy (MBE). After annealing at $T_{a}=400^{\circ}-800^{\circ} \mathrm{C} \mathrm{LT} \mathrm{GaAs} \mathrm{exhibits} \mathrm{an}$ extraordinary high resistance in conjunction with a relatively high photocarrier mobility. ${ }^{1}$ Important for $\mathrm{THz}$ devices are ultrashort carrier lifetimes in LT GaAs, which can be tailored into the sub-picosecond range. ${ }^{2-4}$ All these properties are assigned to the large excess of As $(1 \%-2 \%)$ incorporated during the growth process. In postgrowth annealed LT GaAs, As precipitates of a few $\mathrm{nm}$ in diameter nucleate. ${ }^{5}$ The density of precipitates and point defects can be tailored with $T_{g}$ and $T_{a}$ and hence the electronic and optic properties can differ largely as a function of these temperatures. Two competing models have been proposed for explaining the properties of LT GaAs. In the defect model, EL2-like point defects stemming from excess As play the most important role. ${ }^{6}$ The alternative model assigns the properties of LT GaAs to the formation of As-clusters in a buried Schottkybarrier model. ${ }^{7}$ Ultrashort carrier lifetimes are attributed to either trapping in point defects or in As clusters.

Presently, LT GaAs is used in photomixing, ${ }^{8}$ ultrafast photoconductive switches or substrate material for $\mathrm{THz}$ antennas. ${ }^{9,10}$ For an optimum performance of these devices the lifetime of photogenerated carriers is crucial. The device bandwidth should be closely related to $1 / \tau$, where $\tau$ is the lifetime of mobile carriers. However, a distinct difference is often observed between $\tau$ and the device bandwidth, which can be more than a factor of 2 smaller than $1 / \tau$. On the one hand, this difference certainly is influenced by the capacity and the impedance matching of the device, on the other hand carriers trapped in shallow potentials may be reexcited thermally or by the $\mathrm{THz}$ electric fields, thus reducing the optimum performance concerning bandwidth and noise.

In this letter, we carefully inspect the dynamics of photogenerated carriers close to the band edge in thin LT GaAs

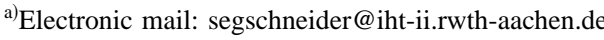

films. Two color time-resolved transmission changes allow to resolve the carrier relaxation close to the band edge with high energetic and temporal resolution. We obtain information on shallow bound sub-band-gap states and point out their relevance for the optoelectronic properties of LT GaAs.

Pump-probe experiments are performed with 50 fs pulses generated from a mode locked Ti:sapphire laser with a spectral width of about $40 \mathrm{meV}$ at a repetition rate of 80 MHz. We use either a conventional lock-in amplifier technique, where the time delay between pump and probe pulses is achieved via a slow stepper motor, or alternatively a fast scanning measurement technique, where the time delay between pump and probe pulse is varied via a retroreflector mounted onto a shaker operating at $70 \mathrm{~Hz}$. Single-line experiments are performed with the full spectral width of pump and probe pulses centered at the same wavelength. For two color pump-probe experiments the pump pulse is spectrally filtered to $10 \mathrm{meV}$ width ( $\sim 200 \mathrm{fs}$ duration). The probe pulse is transmitted unfiltered through the sample, thus not reducing the time resolution. Energy resolved information is obtained by filtering the transmitted probe pulse with a monochromator of approximatly $2.5 \mathrm{meV}$ spectral width and subsequent detection by a photomultiplier. All measurements are performed at $8 \mathrm{~K}$ in order to suppress thermionic emission from shallow bound states.

The LT GaAs samples are grown by MBE at a growth temperature of $200{ }^{\circ} \mathrm{C}$ with a beam equivalent pressure ratio of Ga-to-As $\mathrm{A}_{4}$ of 6:4. The LT films are deposited on a $500 \mathrm{~nm}$ AlAs layer grown at high temperature on (100) GaAs. Postgrowth anneal is performed at $600{ }^{\circ} \mathrm{C}$ for $10 \mathrm{~min}$ in the growth chamber. Thin LT GaAs films are produced by an epitaxial liftoff and are mounted on a sapphire substrate by van der Waals bonding. ${ }^{10,11}$ We note that the observations reported here may not apply to LT GaAs grown or annealed under strongly different conditions. The growth/anneal conditions for our samples were optimized for minimum decay times.

The inset of Fig. 1 shows a cw absorption spectrum of LT GaAs at $8 \mathrm{~K}$. In comparison to high-temperature grown (HT) GaAs the band edge is significantly smeared out and sub-band-gap states up to $40 \mathrm{meV}$ below the unperturbed 


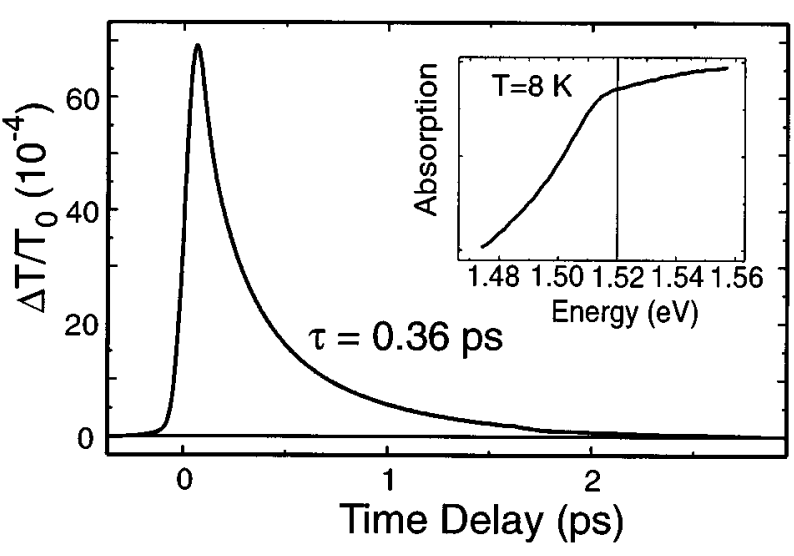

FIG. 1. Single-line pump-probe transients at $1.545 \mathrm{eV}$ central frequency and $8 \mathrm{~K}$. The signal decays with a time constant of $\tau=0.36 \mathrm{ps}$. The inset shows the measured cw absorption spectra of LT GaAs.

band edge of HT GaAs at $1.52 \mathrm{eV}$ are clearly observed. The absorption of these sub-band-gap states is reminiscent of the Urbach slope attributed to a partially broken translational symmetry of the crystal. ${ }^{12}$ Another possible origin of the the red-shifted cw absorption is the Franz-Keldysh effect due to internal Schottky fields associated with As clusters. ${ }^{4}$ A distinction of these two possible origins of the absorption tail will be discussed later. Even at low temperature no sharp excitonic features are resolved in the cw spectra in comparison to HT GaAs. Possible reasons are a large inhomogeneous distribution of excitonic transitions associated with potential fluctuations, strong internal field inhomogeneities, and the ultrashort carrier lifetime.

To determine the optical response time of the sample we first perform single line pump-probe-measurements, which are commonly used for the determination of free carrier lifetimes in LT GaAs. ${ }^{3,13}$ A curve obtained with the fastscanning technique for a central laser energy of $1.545 \mathrm{eV}$, i.e., $25 \mathrm{meV}$ above the band edge at $8 \mathrm{~K}$, is shown in Fig. 1 . The excitation density is $5 \times 10^{17} \mathrm{~cm}^{-3}$. The decay time of $(360 \pm 20)$ fs is derived from a numerical fit to the data. The amplitude of the transmission change is nearly $1 \%$, which is as large as nonlinear transmissions changes obtained in HT GaAs at comparable film thickness under excitation of the excitonic resonance at low temperature and at comparable excitation densities.

In Fig. 2 two-color time-resolved pump-probe signals are shown for the same sample for different probe energies. The interference filter for the pump pulse is set to a central energy of $1.540 \mathrm{eV}$, which is $20 \mathrm{meV}$ above the band gap of HT GaAs. The excitation density is $5 \times 10^{16} e-h$ pairs per $\mathrm{cm}^{3}$, which is well below values reported for trap-saturation effects. ${ }^{14}$ All data are corrected to the average probe intensity at the specific energy. Transmission changes are detectable down to $30 \mathrm{meV}$ below the unperturbed GaAs band edge. Larger detuning energies cannot be achieved due to the limited spectrum of the laser pulse.

There are several remarkable features in the data. All signals decay to zero within a few picoseconds. The transmission change has a minimum close to the pump energy, while a maximum is observed at an energy of $1.50 \mathrm{eV}$, which is $45 \mathrm{meV}$ below the pump energy and $20 \mathrm{meV}$ below the

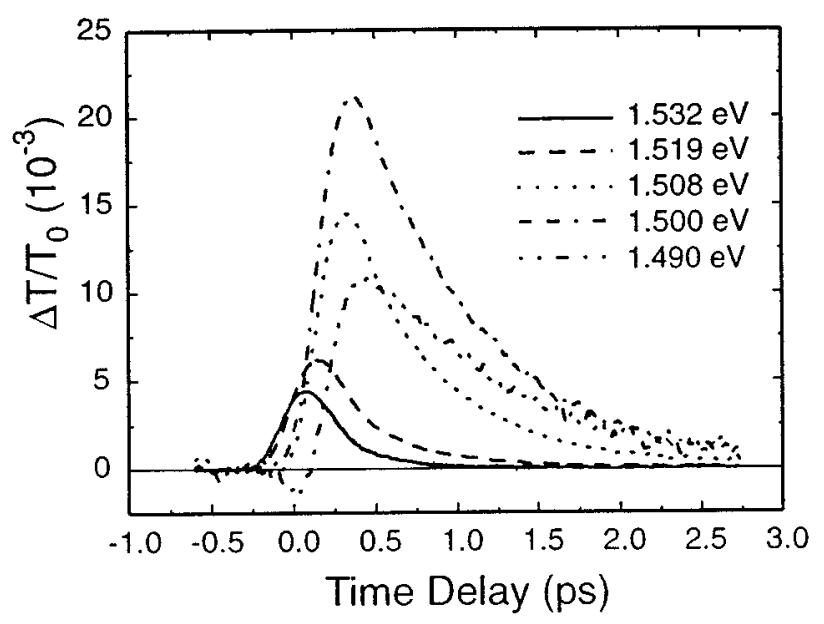

FIG. 2. Energy resolved transient transmission changes for a pump energy of $1.540 \mathrm{eV}$ and various probe energies at $8 \mathrm{~K}$.

unperturbed GaAs band edge. A transmission change of more than $2 \%$ is surprisingly large and comparable to the transmission change obtained at excitonic transitions in quantum wells. ${ }^{15}$ It has to be pointed out, that this transmission change occurs at energies, where the static absorption of the sample has already decreased by $50 \%$ compared to the band edge absorption. In addition to the probe energy dependence of the signal amplitude a distinct difference in the shape of the signals is observed. For probe energies close to the pump pulse, the signal reaches its maximum close to zero time delay, while for smaller probe energies the maximum is shifted towards positive time delays. ${ }^{16}$ Another salient feature in Fig. 2 is that the observed decay times strongly depend on the probe energy. The decay time increases linearly with decreasing probe energy from $\approx(250 \pm 10)$ fs close to the pump energy to $(900 \pm 20)$ fs at $1.490 \mathrm{eV}$, i.e., $30 \mathrm{meV}$ below the bandgap. The time constants below the pump energy are more than a factor of 2 larger than those derived from the single-line measurements. The signal decay is monoexponential for all sub-band-gap energies.

In Fig. 3 the observations are summarized by plotting the temporal shift of the maximum and the decay time constants versus the probe energy. The occurrence of the temporal shift and the detection of a signal at an energy corresponding to

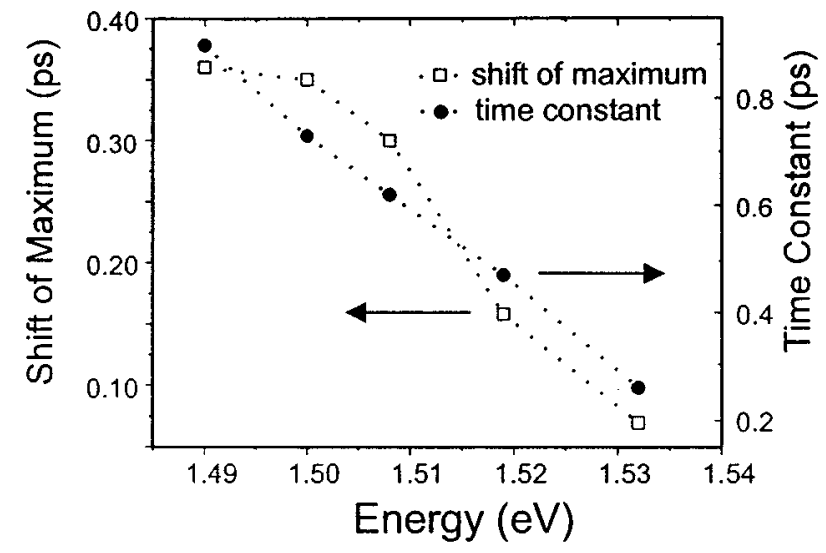

FIG. 3. Temporal shift of the maximum transmission change and time constant vs the probe energy. 
the sub-band-gap transition of HT GaAs reflect the relaxation of hot carriers into sub-band-gap states. The observed dynamics are attributed to electron dynamics due to the smaller density of states in the conduction band. ${ }^{17}$ Obviously, a large amount of hot carriers is able to relax into the sub-band-gap states before they are trapped into deep levels. This points towards a two-step relaxation process rather than a direct relaxation of above band-gap states into the final trapping centers, which are presumably EL2 like midgap states. ${ }^{18}$ Since the initial excess energy relative to the subband-gap states is more than one LO-phonon energy of 36 $\mathrm{meV}$, the relaxation by LO-phonon emission into these states is most probable. The observed time is close to the phonon emission time of $170 \mathrm{fs}$ in GaAs, where we have to take into consideration that momentum selection rules for electronphonon interaction are relaxed in the presence of disorder. From the monoexponential decays of all signals for belowgap probe energies we conclude that the signal decay is dominated by carrier trapping out of the observed states into deep levels rather than transitions within the inhomogeneous distribution of sub-band-gap states. For above band-gap energies an additional fast decay time is observed, which is due to energy relaxation. The linear increase of the time constants for decreasing probe energies is attributed to a decrease in the cross section for trapping in midgap defect levels, when the localization in the sub-band-gap states is concomitantly increasing.

The most probable origin for the observed states are spatial potential fluctuations due to point defects. This interpretation is confirmed by the high optical nonlinearities associated with these states similar to confinement effects in quantum wells. The requirement for observing increased optical nonlinearities is a localization of the wavefunction due to potential fluctuations. For the optical excitation conditions shown here, i.e., excitation of extendend above-gap states, the relaxation into localized sub-band-gap states requires the discussed energy relaxation plus eventually spatial transport. The latter may also contribute to the observed delay in the occupation of these states.

The observed dynamics do not confirm that internal electric Schottky fields are the origin of the sub-band-gap tail of the cw absorption as well as the origin of the localization. ${ }^{7}$ Optical excitation within the Schottky field would lead to a partial screening of electric fields. The associated electroabsorption changes could give rise to positive transmission changes below the band gap as observed. However, the transmission changes should occur approximately at equal time delays for all sub-band-gap energies and not-as observed-with a temporal delay for decreasing energies. Another possible effect is the localization of electrons within the randomly distributed local minima produced by the internal electric fields between the As clusters. For such local minima, the dominant nonlinear absorption change is expected at above-band-gap energies of the partially quantized levels, in contrast to the observed large below band-gap nonlinearity.

A distinct difference in the free carrier lifetimes determined from the single-line and two-color pump-probe experiments is observed. This arises from the fact, that in the single-line experiment mainly the dynamics of the initially optically coupled states dominate the signal. The decay time of these states is mostly dominated by carrier relaxation to lower lying states than by direct trapping into deep levels. The discrepancy in the lifetimes demonstrate that even for very low excess energies in the single-line experiments, the trapping dynamics from states close beneath the band gap may be significantly longer.

The dynamics of the sub-bandgap states have important consequences for the responsivity of $\mathrm{THz}$ devices., e.g., the bandwidth of LT GaAs based $\mathrm{THz}$ antennas is expected to be in the range of $4-5 \mathrm{THz}$, assuming a time constant of $0.36 \mathrm{ps}$ derived from the single-line experiments (Fig. 1). In contrast to this expectation we observed that antennas made from the LT GaAs investigated reach a bandwidth of only $2.5 \mathrm{THz}$ ( $-40 \mathrm{~dB}$ sensitivity). Very likely, the increase of lifetime for carriers in sub-band-gap states affects the performance of LT GaAs based antennas. Additionally, the re-excitation of weakly bound carriers by $\mathrm{THz}$ radiation may increase the noise of antennas.

In conclusion we identify the relevance of sub-band-gap states in a two-step relaxation process of photogenerated carriers in LT GaAs, which exhibit increasing lifetimes with decreasing energy. Potential fluctuations due to the excess As in LT GaAs are probably responsible for the states. The fact that a large optical nonlinearity associated with these states occur at energies, where the linear absorption is low, is attractive for ultrafast modulators and saturable absorbers.

We thank H. M. Heiliger for the epitaxial liftoff films and M. Vossebürger for the characterization of $\mathrm{THz}$ antennas made from the investigated material. This work is supported by the Volkswagen Foundation.

${ }^{1}$ F. W. Smith, A. R. Calawa, C. L. Chen, M. J. Manfra, and L. J. Mahoney, IEEE Electron Device Lett. 9, 77 (1988).

${ }^{2}$ X.-Q. Zhou, H. M. van Driel, W. W. Rühle, Z. Gogolak, and K. Ploog, Appl. Phys. Lett. 61, 3020 (1992).

${ }^{3}$ S. Gupta, M. Y. Frankel, J. A. Valdmanis, J. F. Whitaker, G. A. Mourou, F. W. Smith, and A. R. Calawa, Appl. Phys. Lett. 59, 3276 (1991).

${ }^{4}$ D. D. Nolte, M. R. Melloch, J. M. Woodall, and S. J. Ralph, Appl. Phys. Lett. 62, 1356 (1993).

${ }^{5}$ E. S. Harmon, M. R. Melloch, J. M. Woodall, D. D. Nolte, N. Otsuka, and C. L. Chang, Appl. Phys. Lett. 63, 2248 (1993).

${ }^{6}$ D. C Look, D. C. Walters, M. O. Manasreh, J. R. Sizelove, C. E. Stutz, and K. R. Evans, Phys. Rev. B 42, 3578 (1990).

${ }^{7}$ A. Warren, J. M. Woodall, J. L. Freeouf, D. Grischkowsky, D. T. McInturff, M. R. Melloch, and N. Otsuka, Appl. Phys. Lett. 57, 1331 (1990).

${ }^{8}$ E. R. Brown, K. A. McIntosh, K. B. Nichols, and C. L. Dennis, Appl. Phys. Lett. 66, 285 (1995).

${ }^{9}$ C. Ludwig and J. Kuhl, Appl. Phys. Lett. 69, 1194 (1996).

${ }^{10}$ H.-M. Heiliger, M. Vossebürger, H. G. Roskos, H. Kurz, R. Hey, and K. Ploog, Appl. Phys. Lett. 69, 2903 (1996).

${ }^{11}$ E. Yablonowitch, D. M. Hwang, T. J. Gmitter, L. T. Florez, and J. P. Harbison, Appl. Phys. Lett. 56, 2419 (1990).

${ }^{12}$ S. John and C. H. Grein, Rev. Solid State Sci. 4, 1 (1990).

${ }^{13}$ U. D. Keil, J. M. Hvam, S. Tauz, S. U. Dankowski, P. Kneisel, and G. H. Döhler, Appl. Phys. Lett. 70, 72 (1997).

${ }^{14}$ T. S. Sosnowski, T. B. Norris, H. H. Wang, P. Grenier, J. F. Whitaker, C. Y. Sung, in Ultrafast Electronics and Optoelectronics Technical Digest (Optical Society of America, Washington DC, 1997), pp. 198-200.

${ }^{15}$ Similarily large transmission changes have been observed in the comparison of cw electro-absorption changes in LT AlGaAs and GaAs quantum wells in Ref. 4.

${ }^{16} \mathrm{We}$ ruled out that the temporal delay of the maximum is due to chirp of the probe pulse.

${ }^{17}$ R. Tommasi, P. Langot, and F. Vallee, Appl. Phys. Lett. 66, 1361 (1995).

${ }^{18}$ M. Leszczynski, Phys. Rev. B 53, 10699 (1995). 